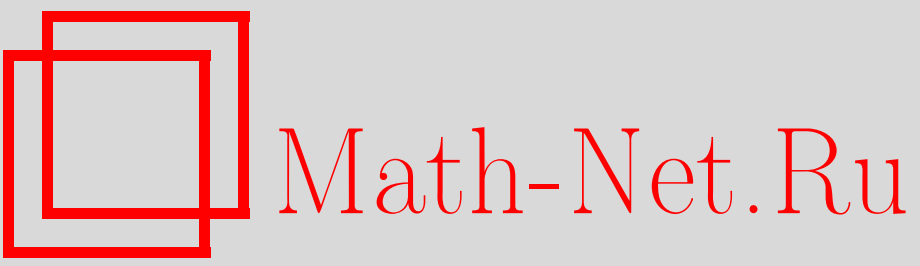

Ю. Ф. Долгий, Р. И. Шевченко, Аппроксимации в задаче устойчивости линейных периодических систем с последействием, Итоги науки и техн. Сер. Соврем. мат. и ее прил. Темат. обз., 2021, том 191, 29-37

DOI: https://doi.org/10.36535/0233-6723-2021-191-29-37

Использование Общероссийского математического портала Math-Net.Ru подразумевает, что вы прочитали и согласны с пользовательским соглашением

http: //www.mathnet.ru/rus/agreement

Параметры загрузки:

IP: 35.174 .16 .151

26 апреля 2023 г., 02:46:26 
ИТОГИ НАУКИ И ТЕХНИКИ.

Современная математика и ее приложения.

Тематические обзоры.

Том 191 (2021). C. $29-37$

DOI: 10.36535/0233-6723-2021-191-29-37

УДК 517.929

\title{
АППРОКСИМАЦИИ В ЗАДАЧЕ УСТОЙЧИВОСТИ ЛИНЕЙНЫХ ПЕРИОДИЧЕСКИХ СИСТЕМ С ПОСЛЕДЕЙСТВИЕМ
}

\author{
(c) 2021 г. . Ф. ДОЛГИЙ, Р. И. ШЕВЧЕНКО
}

\begin{abstract}
АннотАция. Асимптотическая устойчивость линейной периодической системы дифференциальных уравнений с последействием определяется расположением спектра бесконечномерного вполне непрерывного оператора монодромии. Аналитическое представление такого оператора удается получить только для систем специального вида. В численных методах используются конечномерные аппроксимации оператора монодромии. В работе исследуется предложенная Н. Н. Красовским процедура аппроксимации системы дифференциальных уравнений с последействием системами обыкновенных дифференциальных уравнений большой размерности. В гильбертовом пространстве состояний периодической системы с последействием построены конечномерные аппроксимации для оператора монодромии. Доказана теорема, что при росте размерности конечномерных приближений точность аппроксимации оператора монодромии увеличивается.
\end{abstract}

Ключевые слова: система с последействием, устойчивость движения, конечномерная аппроксимация.

\section{APPROXIMATIONS IN THE STABILITY PROBLEM FOR LINEAR PERIODIC SYSTEMS WITH AFTEREFFECT}

\author{
(c) 2021 YU. F. DOLGII, R. I. SHEVCHENKO
}

\begin{abstract}
The asymptotic stability of a linear periodic system of differential equations with aftereffect is determined by the location of the spectrum of the infinite-dimensional, compact monodromy operator. Analytical representations of such operators can be obtained only for systems of a special type. In numerical simulations, finite-dimensional approximations of the monodromy operators are used. In this paper, we examine a procedure for approximating a system of differential equations with aftereffect by systems of ordinary differential equations of large dimension proposed by N. N. Krasovskii. Finitedimensional approximations for monodromy operators are constructed in the Hilbert space of states of a periodic system with aftereffect. We prove that increasing of the dimension of finite-dimensional approximations leads to increasing of the approximation accuracy.
\end{abstract}

Keywords and phrases: system with aftereffect, stability of motion, finite-dimensional approximation.

AMS Subject Classification: 39B82

1. Введение. Статья посвящена развитию теории устойчивости для функционально-дифференциальных уравнений (см. [10]). Объект исследования описывается линейной периодической системой дифференциальных уравнений с последействием. Условия ее асимптотической устойчивости определяются в терминах спектра оператора монодромии, действующего в сепарабельном гильбертовом пространстве (см. [10]). Абстрактные конструкции построения конечномерных аппроксимаций для вполне непрерывных операторов в гильбертовом пространстве описаны в [2]. 
Они использовались в [3] для построения характеристических уравнений, определяющих спектры операторов монодромии для линейных периодических систем дифференциальных уравнений с последействием.

Для линейных периодических систем обыкновенных дифференциальных уравнений теория устойчивости хорошо разработана (см. [12]). При исследовании устойчивости линейной периодической системы дифференциальных уравнений с последействием в статье используются аппроксимирующие системы линейных обыкновенных дифференциальных уравнений с периодическими коэффициентами, которые являются модификациями аппроксимирующих систем, предложенных Н. Н. Красовским (см. [5]).

Существует несколько способов построения аппроксимирующих систем обыкновенных дифференциальных уравнений. Собственные числа и собственные функции оператора монодромии используются при каноническом разложении линейной периодической системы с последействием (см. $[10,11])$, которое позволяет строить конечномерные аппроксимации периодических систем с последействием. Канонические аппроксимации использовались при нахождении управлений в задаче оптимальной стабилизации периодических систем с последействием (см. $[6,16])$.

В работе по теории управления автономной линейной системой с запаздыванием (см. [5]) Н. Н. Красовский использовал аппроксимационные системы обыкновенных дифференциальных уравнений большой размерности, при построении которых применялась замена звена запаздывания последовательной цепочкой инерциальных звеньев. Такие аппроксимационные системы широко используются в теории управления (см. [13,14]), теории стабилизации (см. [15] и теории дифференциальных игр (см. [7]).

Различные модификации аппроксимационных систем Н. Н. Красовского предложены в $[8,9]$. Они применялись в исследованиях по теории устойчивости, теории нелинейных колебаний, теории стабилизации, теории управления и теории дифференциальных игр.

2. Конечномерные аппроксимации оператора монодромии. Рассматривается линейная периодическая система дифференциальных уравнений с последействием

$$
\frac{d x(t)}{d t}=A_{0}(t) x(t)+A_{1}(t) x(t-\tau)+\int_{-\tau}^{0} A(t, \vartheta) x(t+\vartheta) d \vartheta, \quad t \in \mathbb{R}^{+}=(0,+\infty),
$$

где $x:[-\tau,+\infty) \rightarrow \mathbb{R}^{n}$, матричнозначная функция $A_{0}(\cdot) \omega$-периодична и локально интегрируема по Лебегу, матричнозначная функция $A_{1}(\cdot) \omega$-периодична и непрерывна, матричнозначная функция $A(\cdot, \cdot) \omega$-периодична и непрерывна по первому аргументу, а также интегрируема по Лебегу с квадратом по второму аргументу, $\omega>\tau>0$.

Для системы дифференциальных уравнений с последействием (1) предлагаются аппроксимационные системы обыкновенных дифференциальных уравнений (см. [5,8])

$$
\begin{aligned}
& \frac{d y_{0}^{N}}{d t}=A_{0}(t) y_{0}^{N}+A_{1}(t) y_{N}^{N}+\sum_{i=1}^{N} A_{i}^{N}(t) y_{i}^{N}, \\
& \frac{d y_{i}^{N}}{d t}=\frac{N}{\tau}\left(y_{i-1}^{N}-y_{i}^{N}\right), \quad i=\overline{1, N} .
\end{aligned}
$$

Здесь $y_{i}^{N} \in \mathbb{R}^{n}, i=\overline{0, N}, N \geqslant 1, A_{i}^{N}(\cdot), i=\overline{1, N},-\omega$-периодические матричнозначные функции, определяемые формулами

$$
A_{i}^{N}(t)=\int_{-i \tau / N}^{-(i-1) \tau / N} A(t, \vartheta) d \vartheta, \quad i=\overline{1, N}
$$

Введем функциональное пространство $\mathbb{H}=L_{2}\left([-\tau, 0), \mathbb{R}^{n}\right) \times \mathbb{R}^{n}$ со скалярным произведением

$$
\langle\boldsymbol{x}, \boldsymbol{y}\rangle_{\mathbb{H}}=\boldsymbol{y}^{T}(0) \boldsymbol{x}(0)+\int_{-\Delta}^{0} \boldsymbol{y}^{T}(\vartheta) \boldsymbol{x}(\vartheta) d \vartheta, \quad \boldsymbol{x}, \boldsymbol{y} \in \mathbb{H},
$$


и нормой $\|\boldsymbol{x}\|_{\mathbb{H}}=\langle\boldsymbol{x}, \boldsymbol{x}\rangle_{\mathbb{H}}^{1 / 2}, \boldsymbol{x} \in \mathbb{H}$. Задача Коши для системы (1) равномерно корректна. Оператор монодромии для этой системы вполне непрерывен и определяется формулой

$$
(\boldsymbol{U} \varphi)(\vartheta)=x(\omega+\vartheta, \varphi), \quad \vartheta \in[-\tau, 0], \varphi \in \mathbb{H},
$$

где $x(t, \varphi), t \geqslant-\tau,-$ решение задачи Коши с начальной функцией $\varphi \in \mathbb{H}$ (см. [10]). Всюду далее символом $|\cdot|$ обозначена векторная евклидова норма пространства $\mathbb{R}^{n}$ и согласованная с ней норма матрицы как линейного оператора.

Введем фундаментальные нормированные матрицы $\mathcal{Y}^{N}(t)=\left\{\mathcal{Y}_{i j}^{N}(t)\right\}_{i, j=0}^{N}, t \geqslant 0$, системы обыкновенных дифференциальных уравнений $(2),(3), \mathcal{Y}^{N}(0)=I_{(N+1) n}, I_{(N+1) n}-$ единичная матрица. Рассматривается решение задачи Коши $y^{N}(t, \varphi)=\left\{y_{i}^{N}(t, \varphi)\right\}_{i=0}^{N}=\mathcal{Y}^{N}(t)\left\{y_{i}^{N}(0)\right\}_{i=0}^{N}, t \geqslant 0$, системы (2), (3) со специальными начальными условиями

$$
y_{0}^{N}(0)=\varphi(0), \quad y_{i}^{N}(0)=\frac{N}{\tau} \int_{-i \tau / N}^{-(i-1) \tau / N} \varphi(\vartheta) d \vartheta, \quad 1 \leqslant i \leqslant N .
$$

Конечномерные аппроксимации оператора монодромии определяются формулами

$$
\left(\boldsymbol{U}_{N} \varphi\right)(\vartheta)=y_{0}^{N}(\omega+\vartheta, \varphi)=\mathcal{Y}_{00}^{N}(\omega+\vartheta) \varphi(0)+\frac{N}{\tau} \sum_{j=1}^{N} \mathcal{Y}_{0 j}^{N}(\omega+\vartheta) \int_{-j \tau / N}^{-(j-1) \tau / N} \varphi(s) d s,
$$

$\vartheta \in[-\tau, 0], \varphi \in \mathbb{H}$. В работе доказано следующее утверждение.

Теорема 1. При выполнении условий, наложенных на коэфбиииенты системы (1), последовательность аппроксимирующих операторов сходится $к$ оператору $\boldsymbol{U}$ на $\mathbb{H}$, m.e.

$$
\lim _{N \rightarrow+\infty} \boldsymbol{U}_{N} \varphi=\boldsymbol{U} \varphi, \quad \varphi \in \mathbb{H} .
$$

Используются подходы, предложенные в работах Н. Н. Красовского и А. Б. Куржанского [8]. Так, в [5] рассматривалась автономная система дифференциальных уравнений с запаздыванием, а в [8] рассматривалась неавтономная система с одним переменным запаздыванием.

3. Сходимость аппроксимаций оператора монодромии. Операторы $\boldsymbol{U}_{N}, N \geqslant 1$, определяются неявным образом системой обыкновенных дифференциальных уравнений (2), (3). Справедливо следующее утверждение.

Лемма 1. Компонента $y_{0}^{N}(t, \varphi), t \geqslant 0$, решения задачи Коши для системъ обыкновенных дифберенииальных уравнений (2), (3) со специальными начальными условиями

$$
y_{0}^{N}(0)=\varphi(0), \quad y_{i}^{N}(0)=\frac{N}{\tau} \int_{-i \tau / N}^{-(i-1) \tau / N} \varphi(\vartheta) d \vartheta, \quad 1 \leqslant i \leqslant N
$$

где $\varphi \in \mathbb{H}$ удовлетворлет интегральному уравнению

$$
y_{0}^{N}(t)=\varphi(0)+\int_{-\tau}^{0} \hat{\mathcal{A}}^{N}(t, \vartheta) \varphi(\vartheta) d \vartheta+\int_{0}^{t} \mathcal{A}^{N}(t, s) y_{0}^{N}(s) d s, \quad t \geqslant 0 .
$$

Здесь матричнозначные функиии $\hat{\mathcal{A}}^{N}$ и $\mathcal{A}^{N}$ определяются формулами

$$
\begin{aligned}
& \hat{\mathcal{A}}^{N}(t, \vartheta)=\int_{0}^{t}\left[A_{1}\left(s_{1}\right) \hat{\psi}_{N}^{N}\left(s_{1}, \vartheta\right)+\int_{-\tau}^{0} A\left(s_{1}, \vartheta_{1}\right) \hat{\psi}^{N}\left(s_{1}, \vartheta, \vartheta_{1}\right) d \vartheta_{1}\right] d s_{1}, \quad \vartheta \in[-\tau, 0], t \geqslant 0, \\
& \mathcal{A}^{N}(t, s)=A_{0}(s)+\int_{s}^{t}\left[A_{1}\left(s_{1}\right) \psi_{N}^{N}\left(s_{1}, s\right)+\int_{-\tau}^{0} A\left(s_{1}, \vartheta_{1}\right) \psi^{N}\left(s_{1}, s, \vartheta_{1}\right) d \vartheta_{1}\right] d s_{1}, \quad 0 \leqslant s \leqslant t, t \geqslant 0,
\end{aligned}
$$


где скалярные функиии $\hat{\psi}^{N}$ и $\psi^{N}$ определяются формулами

$$
\begin{aligned}
& \hat{\psi}^{N}\left(t, \vartheta, \vartheta_{1}\right)=\sum_{i=1}^{N} \chi_{[-i \tau / N,-(i-1) \tau / N)}\left(\vartheta_{1}\right) \hat{\psi}_{i}^{N}(t, \vartheta), \quad \vartheta_{1}, \vartheta \in[-\tau, 0], t \geqslant 0, \\
& \psi^{N}\left(t, s, \vartheta_{1}\right)=\sum_{i=1}^{N} \chi_{[-i \tau / N,-(i-1) \tau / N)}\left(\vartheta_{1}\right) \psi_{i}^{N}(t, s), \quad \vartheta_{1} \in[-\tau, 0], 0 \leqslant s \leqslant t, t \geqslant 0,
\end{aligned}
$$

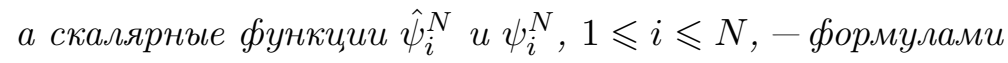

$$
\begin{aligned}
& \hat{\psi}_{i}^{N}(t, \vartheta)=\frac{N}{\tau} \sum_{k=1}^{i} \frac{1}{(i-k) !}\left(\frac{t N}{\tau}\right)^{i-k} \chi_{[-k \tau / N,-(k-1) \tau / N)}(\vartheta) e^{-\frac{N}{\tau} t}, \quad \vartheta \in[-\tau, 0], t \geqslant 0, \\
& \psi_{i}^{N}(t, s)=\frac{1}{(i-1) !}\left(\frac{N}{\tau}\right)^{i}(t-s)^{i-1} e^{-\frac{N}{\tau}(t-s)}, \quad 0 \leqslant s \leqslant t, t \geqslant 0,1 \leqslant i \leqslant N .
\end{aligned}
$$

Для индикатора множества $Е$ используется обозначение $\chi_{E}$.

Доказательство. Учитывая определения матричнозначных функций $A_{i}^{N}, 1 \leqslant i \leqslant N$, систему (2) перепишем в виде

$$
\frac{d y_{0}^{N}(t)}{d t}=A_{0}(t) y_{0}^{N}(t)+A_{1}(t) y_{N}^{N}(t)+\sum_{i=1}^{N} \int_{-\tau}^{0} A\left(t, \vartheta_{1}\right) \chi_{[-i \tau / N,-(i-1) \tau / N)}\left(\vartheta_{1}\right) d \vartheta_{1} y_{i}^{N}(t), \quad t \geqslant 0 .
$$

Используя следующие формулы представления общего решения системы (3) (см. [5])

$$
\begin{gathered}
y_{i}^{N}(t)=\frac{1}{(i-1) !}\left(\frac{N}{\tau}\right)^{i} \int_{0}^{t}(t-s)^{i-1} e^{-\frac{N}{\tau}(t-s)} y_{0}^{N}(s) d s+\sum_{k=1}^{i} \frac{1}{(i-k) !}\left(\frac{t N}{\tau}\right)^{i-k} y_{k}^{N}(0) e^{-\frac{N}{\tau} t}, \\
1 \leqslant i \leqslant N, t \geqslant 0,
\end{gathered}
$$

находим

$$
\begin{aligned}
& \sum_{i=1}^{N} \chi_{[-i \tau / N,-(i-1) \tau / N)}\left(\vartheta_{1}\right) y_{i}^{N}(t)=\int_{0}^{t} \sum_{i=1}^{N} \chi_{[-i \tau / N,-(i-1) \tau / N)}\left(\vartheta_{1}\right) \psi_{i}^{N}(t, s) y_{0}^{N}(s) d s+ \\
& +\int_{-\tau}^{0} \sum_{i=1}^{N} \chi_{[-i \tau / N,-(i-1) \tau / N)}\left(\vartheta_{1}\right) \hat{\psi}_{i}^{N}(t, \vartheta) \varphi(\vartheta) d \vartheta=\int_{0}^{t} \psi^{N}\left(t, s, \vartheta_{1}\right) y_{0}^{N}(s) d s+\int_{-\tau}^{0} \hat{\psi}^{N}\left(t, \vartheta, \vartheta_{1}\right) \varphi(\vartheta) d \vartheta, \\
& \vartheta_{1} \in[-\tau, 0], t \geqslant 0,1 \leqslant i \leqslant N .
\end{aligned}
$$

В результате равенство (5) преобразуется к виду

$$
\begin{aligned}
& \frac{d y_{0}^{N}(t)}{d t}=A_{0}(t) y_{0}^{N}(t)+A_{1}(t) {\left[\int_{0}^{t} \psi_{N}^{N}(t, s) y_{0}^{N}(s) d s+\int_{-\tau}^{0} \hat{\psi}_{N}^{N}(t, \vartheta) \varphi(\vartheta) d \vartheta\right]+} \\
&+\int_{-\tau}^{0} A\left(t, \vartheta_{1}\right)\left[\int_{0}^{t} \psi^{N}\left(t, s, \vartheta_{1}\right) y_{0}^{N}(s) d s+\int_{-\tau}^{0} \hat{\psi}^{N}\left(t, \vartheta, \vartheta_{1}\right) \varphi(\vartheta) d \vartheta\right] d \vartheta_{1}= \\
&=A_{0}(t) y_{0}^{N}(t)+\int_{0}^{t}\left[A_{1}(t) \psi_{N}^{N}(t, s)+\int_{-\tau}^{0} A\left(t, \vartheta_{1}\right) \psi^{N}\left(t, s, \vartheta_{1}\right) d \vartheta_{1}\right] y_{0}^{N}(s) d s+ \\
&+\int_{-\tau}^{0}\left[A_{1}(t) \hat{\psi}_{N}^{N}(t, \vartheta)+\int_{-\tau}^{0} A\left(t, \vartheta_{1}\right) \hat{\psi}^{N}\left(t, \vartheta, \vartheta_{1}\right) d \vartheta_{1}\right] \varphi(\vartheta) d \vartheta, \quad t \geqslant 0 .
\end{aligned}
$$


Интегрируя полученное равенство, находим интегральное уравнение

$$
\begin{aligned}
y_{0}^{N}(t) & =\varphi(0)+\int_{-\tau}^{0} \int_{0}^{t}\left[A_{1}(s) \hat{\psi}_{N}^{N}(s, \vartheta)+\int_{-\tau}^{0} A\left(s, \vartheta_{1}\right) \hat{\psi}^{N}\left(s, \vartheta, \vartheta_{1}\right) d \vartheta_{1}\right] d s \varphi(\vartheta) d \vartheta+ \\
& +\int_{0}^{t}\left\{A_{0}(s)+\int_{s}^{t}\left[A_{1}\left(s_{1}\right) \psi_{N}^{N}\left(s_{1}, s\right)+\int_{-\tau}^{0} A\left(s_{1}, \vartheta_{1}\right) \psi^{N}\left(s_{1}, s, \vartheta_{1}\right) d \vartheta_{1}\right] d s_{1}\right\} y_{0}^{N}(s) d s, \quad t \geqslant 0 .
\end{aligned}
$$

Лемма доказана.

Аппроксимирующие операторы $\boldsymbol{U}_{N}, N \geqslant 1$, определяются интегральными уравнениями (4) неявным образом. Оператор $\boldsymbol{U}$ также определим неявным образом с помощью интегрального уравнения. Докажем лемму.

Лемма 2. Решение задачи Коши для системъ (1) с начальной функиией $\varphi \in \mathbb{H}$ удовлетворяет интегральному уравнению

$$
x(t)=\varphi(0)+\int_{-\tau}^{0} \hat{\mathcal{A}}(t, \vartheta) \varphi(\vartheta) d \vartheta+\int_{0}^{t} \mathcal{A}(t, s) x(s) d s, \quad t \geqslant 0
$$

где матричнозначные функиии $\hat{\mathcal{A}}$ и $\mathcal{A}$ определяются формулами

$$
\begin{aligned}
& \hat{\mathcal{A}}(t, \vartheta)=\chi_{[-\tau, t-\tau]}(\vartheta) A_{1}(\vartheta+\tau)+\int_{-\tau}^{0} \chi_{\left[\vartheta_{1}, t+\vartheta_{1}\right]}(\vartheta) A\left(\vartheta-\vartheta_{1}, \vartheta_{1}\right) d \vartheta_{1}, \quad \vartheta \in[-\tau, 0], t \geqslant 0, \\
& \mathcal{A}(t, s)=A_{0}(s)+\chi_{[-\tau, t-\tau]}(s) A_{1}(s+\tau)+\int_{-\tau}^{0} \chi_{\left[\vartheta_{1}, t+\vartheta_{1}\right]}(s) A\left(s-\vartheta_{1}, \vartheta_{1}\right) d \vartheta_{1}, \quad 0 \leqslant s \leqslant t, t \geqslant 0 .
\end{aligned}
$$

Доказательство. Решение задачи Коши для системы (1) удовлетворяет условию $x(t)=\varphi(t)$ при $t \in[-\tau, 0]$ и уравнению

$$
x(t)=\varphi(0)+\int_{0}^{t} A_{0}(s) x(s) d s+\int_{0}^{t} A_{1}(s) x(s-\tau) d s+\int_{0}^{t} \int_{-\tau}^{0} A\left(s_{1}, \vartheta_{1}\right) x\left(s_{1}+\vartheta_{1}\right) d \vartheta_{1} d s_{1}, \quad t \geqslant 0 .
$$

Справедливы равенства

$$
\begin{aligned}
& \int_{0}^{t} A_{1}(s) x(s-\tau) d s=\int_{-\tau}^{t} \chi_{[-\tau, t-\tau]}(s) A_{1}(s+\tau) x(s) d s= \\
& =\int_{-\tau}^{0} \chi_{[-\tau, t-\tau]}(\vartheta) A_{1}(\vartheta+\tau) \varphi(\vartheta) d \vartheta+\int_{0}^{t} \chi_{[-\tau, t-\tau]}(s) A_{1}(s+\tau) x(s) d s, \quad t \geqslant 0, \\
& \int_{0}^{t} \int_{-\tau}^{0} A\left(s_{1}, \vartheta_{1}\right) x\left(s_{1}+\vartheta_{1}\right) d \vartheta_{1} d s_{1}= \\
& =\int_{-\tau}^{0} \int_{\vartheta_{1}}^{t+\vartheta_{1}} A\left(s-\vartheta_{1}, \vartheta_{1}\right) x(s) d s d \vartheta_{1}=\int_{-\tau}^{0} \int_{-\tau}^{t} \chi_{\left[\vartheta_{1}, t+\vartheta_{1}\right]}(s) A\left(s-\vartheta_{1}, \vartheta_{1}\right) x(s) d s d \vartheta_{1}=
\end{aligned}
$$




$$
=\int_{-\tau}^{0} \int_{-\tau}^{0} \chi_{\left[\vartheta_{1}, t+\vartheta_{1}\right]}(\vartheta) A\left(\vartheta-\vartheta_{1}, \vartheta_{1}\right) d \vartheta_{1} \varphi(\vartheta) d \vartheta+\int_{0}^{t} \int_{-\tau}^{0} \chi_{\left[\vartheta_{1}, t+\vartheta_{1}\right]}(s) A\left(s-\vartheta_{1}, \vartheta_{1}\right) d \vartheta_{1} x(s) d s, \quad t \geqslant 0 .
$$

Подстановка этих равенств в (7) завершает доказательство леммы.

Для доказательства сходимости аппроксимирующих операторов используем теорему БанахаШтейнгауза (см. [4, с. 266]).

Лемма 3. Аппроксимирующие операторы монодромии $\boldsymbol{U}_{N}, N \geqslant 1$, равномерно ограниченъ.

Доказательство. Представим уравнение (4) в операторной форме

$$
y_{0}^{N}(t)=\left(\boldsymbol{K}_{N} \varphi\right)(t)+\left(\boldsymbol{V}_{N} y_{0}^{N}\right)(t), \quad t \in[0, \omega], \varphi \in \mathbb{H},
$$

где операторы $\boldsymbol{K}_{N}$ и $\boldsymbol{V}_{N}$ определяются формулами

$$
\begin{gathered}
\left(\boldsymbol{K}_{N} \varphi\right)(t)=\varphi(0)+\int_{-\tau}^{0} \hat{\mathcal{A}}^{N}(t, \vartheta) \varphi(\vartheta) d \vartheta, \quad 0 \leqslant t \leqslant \omega, \varphi \in \mathbb{H}, \\
\left(\boldsymbol{V}_{N} y_{0}^{N}\right)(t)=\int_{0}^{t} \mathcal{A}^{N}(t, s) y_{0}^{N}(s) d s, \quad 0 \leqslant t \leqslant \omega .
\end{gathered}
$$

Здесь $\boldsymbol{K}_{N}: \mathbb{H} \rightarrow C\left([0, \omega], \mathbb{R}^{n}\right)$ - конечномерный оператор, $\boldsymbol{V}_{N}: C\left([0, \omega], \mathbb{R}^{n}\right) \rightarrow C\left([0, \omega], \mathbb{R}^{n}\right)$ вольтерров оператор.

Имеет место неравенство

$$
\sup _{t \in[0, \omega]}\left|\left(\boldsymbol{K}_{N} \varphi\right)(t)\right| \leqslant|\varphi(0)|+\int_{-\tau}^{0} \sup _{t \in[0, \omega]}\left|\hat{\mathcal{A}}^{N}(t, \vartheta)\right||\varphi(\vartheta)| d \vartheta
$$

где

$$
\sup _{t \in[0, \omega]}\left|\hat{\mathcal{A}}^{N}(t, \vartheta)\right| \leqslant \int_{0}^{\omega}\left[\left|A_{1}\left(s_{1}\right)\right| \hat{\psi}_{N}^{N}\left(s_{1}, \vartheta\right)+\int_{-\tau}^{0}\left|A\left(s_{1}, \vartheta_{1}\right)\right| \hat{\psi}^{N}\left(s_{1}, \vartheta, \vartheta_{1}\right) d \vartheta_{1}\right] d s_{1}, \quad \vartheta \in[-\tau, 0] .
$$

Учитывая определения функций $\hat{\psi}_{N}^{N}$ и $\hat{\psi}^{N}$, можно показать, что существует функция $\alpha(\cdot) \in$ $L_{2}[-\tau, 0]$, для которой выполняется оценка

$$
\sup _{t \in[0, \omega]}\left|\hat{\mathcal{A}}^{N}(t, \vartheta)\right| \leqslant \alpha(\vartheta), \quad \vartheta \in[-\tau, 0] .
$$

В результате находим

$$
\begin{array}{r}
\sup _{t \in[0, \omega]}\left|\left(\boldsymbol{K}_{N} \varphi\right)(t)\right| \leqslant|\varphi(0)|+\left(\int_{-\tau}^{0} \alpha^{2}(\vartheta) d \vartheta\right)^{1 / 2}\left(\int_{-\tau}^{0}|\varphi(\vartheta)|^{2} d \vartheta\right)^{1 / 2} \\
\leqslant \sqrt{2} \max \left\{1,\left(\int_{-\tau}^{0} \alpha^{2}(\vartheta) d \vartheta\right)^{1 / 2}\right\}\|\varphi\|_{\mathbb{H}} .
\end{array}
$$

Таким образом, операторы $\boldsymbol{K}_{N}: \mathbb{H} \rightarrow C\left([0, \omega], \mathbb{R}^{n}\right)$ равномерно ограничены по $N$.

Из определения функций $\psi_{N}^{N}$ и $\psi^{N}$ следует, что существуют такие ограниченные функции $\beta_{1}$ и $\beta_{2}$, для которых справедливы неравенства $\left|\psi_{N}^{N}\left(s_{1}, s\right)\right| \leqslant \beta_{1}\left(s_{1}, s\right)$ и $\left|\psi^{N}\left(s_{1}, s, \vartheta_{1}\right)\right| \leqslant \beta_{2}\left(s_{1}, s, \vartheta_{1}\right)$, $s \leqslant s_{1} \leqslant t,-\tau \leqslant \vartheta_{1} \leqslant 0,0 \leqslant s \leqslant t, 0 \leqslant t \leqslant \omega$. Отсюда находим

$$
\left|\mathcal{A}^{N}(t, s)\right| \leqslant\left|A_{0}(s)\right|+\int_{s}^{t}\left[\left|A_{1}\left(s_{1}\right)\right| \beta_{1}\left(s_{1}, s\right)+\int_{-\tau}^{0}\left|A\left(s_{1}, \vartheta_{1}\right)\right| \beta_{2}\left(s_{1}, s, \vartheta_{1}\right) d \vartheta_{1}\right] d s_{1},
$$


$0 \leqslant s \leqslant t, 0 \leqslant t \leqslant \omega, N \geqslant 1$. Из приведенных оценок следует, что операторы

$$
\left(\boldsymbol{I}-\boldsymbol{V}_{N}\right)^{-1}: C\left([0, \omega], \mathbb{R}^{n}\right) \rightarrow C\left([0, \omega], \mathbb{R}^{n}\right), \quad\left(\boldsymbol{I}-\boldsymbol{V}_{N}\right)^{-1}=\sum_{k=0}^{\infty} \boldsymbol{V}_{N}^{k},
$$

равномерно ограничены по $N$. Следовательно, операторы $\left(\boldsymbol{I}-\boldsymbol{V}_{N}\right)^{-1} \boldsymbol{K}_{N}: \mathbb{H} \rightarrow C\left([0, \omega], \mathbb{R}^{n}\right)$ также равномерно ограничены поЁ $N$. Аппроксимирующие операторы монодромии $\boldsymbol{U}_{N}$ определяются формулами

$$
\left(\boldsymbol{U}_{N} \varphi\right)(\vartheta)=\left(\left(\boldsymbol{I}-\boldsymbol{V}_{N}\right)^{-1} \boldsymbol{K}_{N} \varphi\right)(\omega+\vartheta), \quad \vartheta \in[-\tau, 0], \varphi \in \mathbb{H} .
$$

Области значений этих операторов всюду плотны в пространстве $\mathbb{H}$, что завершает доказательство леммы.

Запишем интегральное уравнение (6) в операторной форме

$$
x(t)=(\boldsymbol{K} \varphi)(t)+(\boldsymbol{V} x)(t), \quad t \in[0, \omega],
$$

где операторы $\boldsymbol{K}$ и $\boldsymbol{V}$ определяются формулами

$$
\begin{gathered}
(\boldsymbol{K} \varphi)(t)=\varphi(0)+\int_{-\tau}^{0} \hat{\mathcal{A}}(t, \vartheta) \varphi(\vartheta) d \vartheta, \quad 0 \leqslant t \leqslant \omega, \varphi \in \mathbb{H}, \\
(\boldsymbol{V} x)(t)=\int_{0}^{t} \mathcal{A}(t, s) x(s) d s, \quad 0 \leqslant t \leqslant \omega .
\end{gathered}
$$

Здесь $\boldsymbol{K}: \mathbb{H} \rightarrow C\left([0, \omega], \mathbb{R}^{n}\right), \boldsymbol{V}: C\left([0, \omega], \mathbb{R}^{n}\right) \rightarrow C\left([0, \omega], \mathbb{R}^{n}\right)$ - вольтерров оператор. Оператор монодромии определяется формулой

$$
(\boldsymbol{U} \varphi)(\vartheta)=\left((\boldsymbol{I}-\boldsymbol{V})^{-1} \boldsymbol{K} \varphi\right)(\omega+\vartheta), \quad \vartheta \in[-\tau, 0], \varphi \in \mathbb{H} .
$$

Для доказательства теоремы нужно показать, что

$$
\lim _{N \rightarrow+\infty}\left(\boldsymbol{U}_{N}-\boldsymbol{U}\right) \varphi=0, \quad \varphi \in \mathbb{H} .
$$

Имеем

$$
\begin{aligned}
\left(\left(\boldsymbol{I}-\boldsymbol{V}_{N}\right)^{-1} \boldsymbol{K}_{N} \varphi\right)(\omega+\vartheta)-\left((\boldsymbol{I}-\boldsymbol{V})^{-1} \boldsymbol{K} \varphi\right)(\omega+\vartheta) & = \\
& =\left(\left(\boldsymbol{I}-\boldsymbol{V}_{N}\right)^{-1}\left(\boldsymbol{V}_{N}-\boldsymbol{V}\right)(\boldsymbol{I}-\boldsymbol{V})^{-1} \boldsymbol{K}_{N} \varphi\right)(\omega+\vartheta)+\left((\boldsymbol{I}-\boldsymbol{V})^{-1}\left(\boldsymbol{K}_{N}-\boldsymbol{K}\right) \varphi\right)(\omega+\vartheta),
\end{aligned}
$$

$\vartheta \in[-\tau, 0], \varphi \in \mathbb{H}$. Поэтому осталось доказать следующее утверждение.

Лемма 4. Последовательности операторов $\boldsymbol{V}_{N}: C\left([0, \omega], \mathbb{R}^{n}\right) \rightarrow C\left([\omega-\tau, \omega], \mathbb{R}^{n}\right), N \geqslant 1$, u $\boldsymbol{K}_{N}: \mathbb{H} \rightarrow C\left([\omega-\tau, \omega], \mathbb{R}^{n}\right), N \geqslant 1$, сходлтсл, т.е.

$$
\begin{aligned}
\lim _{N \rightarrow+\infty} \boldsymbol{V}_{N} x & =\boldsymbol{V} x, \quad x \in C\left([0, \omega], \mathbb{R}^{n}\right), \\
\lim _{N \rightarrow+\infty} \boldsymbol{K}_{N} \varphi & =\boldsymbol{K} \varphi, \quad \varphi \in \tilde{\mathbb{H}},
\end{aligned}
$$

где $\widetilde{\mathbb{H}}-$ множсество функиий, всюду плотное в $\mathbb{H}$.

Доказательство. Учитывая вид операторов $\boldsymbol{V}_{N}$ и $\boldsymbol{V}$, необходимо показать, что при $t \in[\omega-\tau, \omega]$ справедливо

$$
\lim _{N \rightarrow+\infty} \mathcal{A}^{N}(t, s)=\mathcal{A}(t, s)
$$

при $s$, принадлежащем отрезку $[0, t]$, за исключением множества малой меры. Учитывая непрерывность функции $A_{1}(\cdot)$ и функции $A(\cdot, \cdot)$ по первому аргументу и используя для них модуль 
непрерывности (см. [1, с. 17]), доказательство требуемого утверждения можно свести к обоснованию существования пределов

$$
\begin{gathered}
\lim _{N \rightarrow+\infty} \int_{s}^{t} \psi_{N}^{N}\left(s_{1}, s\right) d s_{1}=\chi_{[-\tau, t-\tau]}(s), \\
\lim _{N \rightarrow+\infty} \int_{s}^{t} \psi^{N}\left(s_{1}, s, \vartheta_{1}\right) d s_{1}=\chi_{\left[\vartheta_{1}, t+\vartheta_{1}\right]}(s),
\end{gathered}
$$

при $t \in[\omega-\tau, \omega], s \in[0, t], \vartheta_{1} \in[-\tau, 0]$, за исключением множества малой меры. Обоснование указанных утверждений приведено в $[5,8]$.

При доказательстве сходимости операторов $\boldsymbol{K}_{N}, N \geqslant 1$, требуется показать, что при $t \in[\omega-$ $\tau, \omega]$ справедливо

$$
\lim _{N \rightarrow+\infty} \hat{\mathcal{A}}^{N}(t, \vartheta)=\hat{\mathcal{A}}(t, \vartheta)
$$

при $\vartheta$, принадлежащем отрезку $[-\tau, 0]$, за исключением множества малой меры. Учитывая непрерывность функции $A_{1}(\cdot)$ и функции $A(\cdot, \cdot)$ по первому аргументу, доказательство требуемого утверждения можно свести к обоснованию существования пределов

$$
\begin{gathered}
\lim _{N \rightarrow+\infty} \int_{0}^{t} \hat{\psi}_{N}^{N}(s, \vartheta) d s=\chi_{[-\tau, t-\tau]}(\vartheta), \\
\lim _{N \rightarrow+\infty} \int_{0}^{t} \hat{\psi}^{N}\left(s, \vartheta, \vartheta_{1}\right) d s=\chi_{\left[\vartheta_{1}, t+\vartheta_{1}\right]}(\vartheta),
\end{gathered}
$$

при $t \in[\omega-\tau, \omega], \vartheta \in[-\tau, 0], \vartheta_{1} \in[-\tau, 0]$, за исключением множества малой меры. Последние утверждения обоснованы в $[5,8]$.

\section{СПИСОК ЛИТЕРАТУРЫ}

1. Бердышев В. И., Субботин Ю. И. Численные методы приближения функций. - Свердловск: СреднеУральское книжное изд-во, 1979.

2. Гохберг И. Ц., Крейн М. Г. Введение в теорию линейных несамосопряженных операторов в гильбертовом пространстве. - М.: Наука, 1965.

3. Долгий Ю. Ф. Характеристическое уравнение в задаче асимптотической устойчивости периодической системы с последействием// Тр. ин-та мат. мех. УрО РАН. - 2005. - 11, № 1. - С. 85-96.

4. Канторович Л. В., Акилов Г. П. Функциональный анализ. - М.: Наука, 1984.

5. Красовский $H$. Н. Об аппроксимации одной задачи аналитического конструирования регуляторов в системе с запаздыванием// Прикл. мат. мех. - 1964. - 28, № 4. - С. 716-724.

6. Красовский Н. Н., Осипов Ю. С. О стабилизации движений управляемого объекта с запаздыванием в системе регулирования// Изв. АН СССР. Техн. киберн. - 1963. - 6. - С. 3-15.

7. Кряжимский А. В., Максимов В. И. Аппроксимация линейных дифференциально-разностных игр// Прикл. мат. мех. - 1978. - 42, № 2. - С. 202-209.

8. Куржанский А. Б. К аппроксимации линейных дифференциальных уравнений с запаздыванием// Диффер. уравн. - 1967. - 3, № 12. - С. 2094-2107.

9. Репин Ю. М. О приближенной замене систем с запаздыванием обыкновенными динамическими системами// Прикл. мат. мех. - 1965. - 29, № 2. - С. 226-235.

10. Хейл Дж. Теория функционально-дифференциальных уравнений. - М.: Мир, 1984.

11. Шиманов C. Н. K теории линейных дифференциальных уравнений с периодическими коэффициентами и запаздыванием времени// Прикл. мат. мех. - 1963. - 27, № 3. - С. 450-458.

12. Якубович B. А., Старжинский B. М. Линейные дифференциальные уравнения с периодическими коэффициентами и их приложения. - М.: Наука, 1972.

13. Burns J. A., Clif E. Methods for approximating solutions to linear hereditary quadratic optimal control problems// IEEE Trans. Automat. Control. — 1978. — 23, № 1. — P. 21-26. 
14. Delfour M. C. The linear quadratic optimal control problem for hereditary differential systems: Theory and numerical solution// SIAM J. Appl. Math. Optim. — 1977. — 3, № 2. — P. 101-162.

15. Gibson J. S. Linear-quadratic optimal control of hereditary differential systems: infinite dimensional Riccati equations and numerical approximations// SIAM J. Control Optim. — 1983. — 21, № 1. — P. 95-139.

16. Pandolf $L$. Stabilization of neutral functional differential equations// J. Optim. Theory Appl. - 1976. 20, № 2. - P. 191-204.

Долгий Юрий Филиппович

Уральский федеральный университет

им. первого Президента России Б. Н. Ельцина, Екатеринбург;

Институт математики и механики им. Н. Н. Красовского

Уральского отделения РАН, Екатеринбург

E-mail: Jury.Dolgy@urfu.ru

Шевченко Роман Иванович

Уральский федеральный университет

им. первого Президента России Б. Н. Ельцина, Екатеринбург

E-mail: oma170@hotmail.com 